\title{
Electrooculogram Signals Analysis for Process Control Operator Based on Fuzzy c-Means
}

\author{
Jiangwen Song \\ School of Electronic and Electrical Engineering \\ Shanghai University of Engineering Science \\ Shanghai, China, 201620
}

\author{
Raofen Wang* \\ School of Electronic and Electrical Engineering \\ Shanghai University of Engineering Science \\ Shanghai, China, 201620
}

\author{
Guanghua Zhang \\ School of Electronic and Electrical Engineering \\ Shanghai University of Engineering Science \\ Shanghai, China, 201620
}

\author{
Chaoxing Xiong \\ School of Electronic and Electrical Engineering \\ Shanghai University of Engineering Science \\ Shanghai, China, 201620 \\ Leyan Zhang \\ School of Electronic and Electrical Engineering \\ Shanghai University of Engineering Science \\ Shanghai, China, 201620 \\ Cunbang Sun \\ School of Electronic and Electrical Engineering \\ Shanghai University of Engineering Science \\ Shanghai, China, 201620
}

\begin{abstract}
Biomedical signals of human can reflect the body's task load, fatigue and other psychological information. Compared with other biomedical signals, electrooculogram (EOG) has higher amplitude, less interference, and is easy to detect. In this paper, the EOG signals of operator's were analyzed. Wavelet transform was used to remove the highfrequency artifacts. Then fuzzy c-means was adopted to detect the eye blink peak points of EOG. After that, eye blink interval (EBI) of operator was calculated. Four EOG features (the average of EBI, variance of EBI, standard deviation of EBI and variation coefficient of EBI) were extracted. Finally, the relationship between EOG features and operator's fatigue, effort, anxiety and task load were analyzed. The experimental results illustrate that EOG features had some relation to the operator's fatigue, effort, anxiety and task load respectively.
\end{abstract}

Keywords-electrooculogram; fuzzy c-means; operator functional state; fatigue

\section{INTRODUCTION}

Fatigue is a natural physiological phenomenon, which is related to the operator's labor intensity, work environment, health, mood and so on. It is a kind of self-adjustment and protection function of human body. In recent years, with the rapid development of economy and the faster pace of life, many of the operators continue to work in the state of fatigue $[1,2]$. Consequently, many accidents occurred because of operator fatigue. And also the anxiety and effort level of the operators can affect their work performance. The operator's performance degradation may be the reason of some serious disasters, particularly in safety-critical applications. The assessment of operator mental workload would help predict the operator's periods of high operational risk. Early warning based on prediction of the operator's mental workload is one of the effective methods to prevent such accidents. Therefore, the accurate assessment of operator mental workload is the key to provide early warning successfully.

Current scientific research found that, the human body contains many types of biological signals which can reflect in parts the operator's fatigue, anxiety, mental workload, etc. [3-5] After comparing the characteristics of electroencephalogram (EEG), electrocardiogram (ECG) and electrooculogram (EOG), the authors analyzed EOG signals of operator's. Features of EOG signals contain eye blink rate, eye blink amplitude, closing time, eye blink variability, etc. [6] The researchers thought that EOG is associated with operator's mental workload because eye blink is associated with facial nerve and the awakening is associated with central nerve. When task load increases, the visual demand is likely to increase which may lead to eye blink rate decrease. Simultaneously, the eye activity may increase. When the operator focuses on the tasks, his/her eyes blink rate may decrease and the eye blink interval may increase $[7,8]$. In $[9,10]$, the authors analyzed the driver's eye blink interval. It is illustrated that when the driver is fatigue, his/her eyelid ptosis arises, eye blink amplitude decreases. In other words eye blink interval decreases while closing time increases.

This paper is to investigate the EOG signals associated with the operator functional state (OFS), like fatigue, anxiety, effort and task load. The experimental environment of process control was designed, and the EOG signals of operators under multi-level of task loads were sampled. Fuzzy c-means (FCM) was adopted to detect the eye blink peak points of EOG. And then EOG features to assess the human fatigue, anxiety, effort and task load were found through the study of EOG. Thereby, these indices will be used to predict the functional state of the operator. Early warning system can be constructed to prevent accidents which are caused by human factors. 
The rest of the paper is arranged as follows. Section 2 described the data acquisition experiment in details. In section 3 , the Fuzzy c-means algorithm is presented. In section 4 , the EOG signal analyzing process and results are illustrated, and the discussions are presented. Section 5 makes the conclusions and gives the future works.

\section{EXPERIMENT}

\section{A. Data Acquisition}

The experiments were carried out on Automation-enhanced cabin air management system (AUTOCAMS), developed by Hockey and his colleagues [11] and modified by Lorenz [12]. AUTOCAMS simulates a life support system of a spacecraft. It requires the subject to manage a semi-automatic system which is to maintain comfortable atmospheric condition of the cabin. There are four sub-systems corresponding to four critical parameters of atmospheric condition (oxygen concentration, carbon dioxide concentration, temperature, pressure). The subject was instructed to control some of the sub-systems manually according to the experimental design.

Subjects were trained on the manual process control tasks for over 7 hours, followed by a further 5 hour experience of controlling the system under a range of conditions. After that, the subjects are regarded as the adept at operating AUTOCAMS. Three subjects (SA, SB, and SC) participated in this experiment.

The task of the subject was defined to control some of the four subsystems so as to maintain their relevant variables within target ranges. There are 8 control conditions $(\mathrm{C} 1 \sim \mathrm{C} 8)$ in the experiment. The first $(\mathrm{C} 1)$ and last $(\mathrm{C} 8)$ conditions are five minute baseline testing conditions in which the subjects' task was to monitor the operation of AUTOCAMS. The amount of manually controlled subsystems in other control conditions $(\mathrm{C} 2 \sim \mathrm{C} 7)$ is $1,3,4,4,3,1$, respectively and each condition lasts for 15 minutes. For an experimental session, the cyclic loading method resulted in a loading phase $(\mathrm{C} 2 \sim \mathrm{C} 4)$ and an unloading phase (C5 C7).

Every subject was required to do the experiment for three times (e.g. SA1, SA2, SA3) at the same time on different days to avoid circadian effect.

\section{B. EOG Data}

The subjects' vertical EOG signals were recorded by Nihon-Koden EEG 1100 physiological recorder from 2 sites, which are placed above and under the subject's right eye and referenced to linked earlobes. The EOG data were sampled at $500 \mathrm{~Hz}$.

\section{METHODS}

\section{A. Fuzzy c-Means}

Fuzzy c-means is a data clustering method in which each data point belongs to a cluster, with a degree specified by a membership grade [13-15]. FCM partitions a collection of $n$ vectors $X=\left\{x_{1}, x_{2}, \cdots, x_{n}\right\}$, where $x_{k}=\left(x_{k 1}, x_{k 2}, \cdots, x_{k s}\right)$ is the $k$ th vector of $X$. FCM is based on minimizing an objective function, which respects to fuzzy membership ' $U$ ', and set of cluster centroids, ' $V$ '. The FCM objective function can be described as,

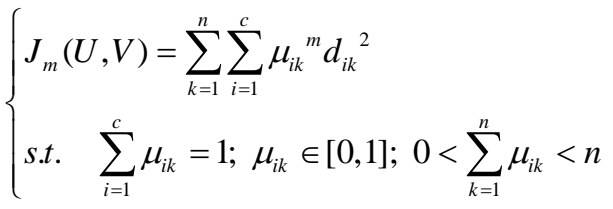

where, ' $c$ ' is the number of clusters. $\mu_{i k}$ is the membership function of vector $x_{k}$ to the $i$ th cluster. $d_{i k}{ }^{2}=\left\|x_{k}-v_{i}\right\|^{2}$ can be either Euclidean distance or one of its generalizations such as Mahalanobis distance. Euclidean distance is used in this study.

The steps of the FCM method are explained in brief:

- Firstly, the centers of each cluster $V(0)$, fuzzy coefficient ' $m$ ', number of cluster ' $c$ ' and termination threshold ' $\varepsilon$ ' are randomly selected.

- Secondly, the membership matrix $U^{(t)}$ is computed with the following equation:

$$
\mu_{i k}(t)=1 / \sum_{j=1}^{c}\left(d_{i k}(t) / d_{j k}(t)\right)^{2 / m-1} .
$$

- Thirdly, the $c$ th cluster centroid is calculated with the following equation:

$$
v_{i}(t+1)=\sum_{k=1}^{n} \mu_{i k}(t)^{m} x_{k} / \sum_{k=1}^{n} \mu_{i k}(t)^{m} .
$$

- The process is stopped if $\left\|V^{(t+1)}-V^{(t)}\right\|<\varepsilon$.

In this paper, the FCM algorithm was used to separate EOG signals into 2 subsets, i.e. the higher amplitude subset and the lower amplitude subset. Then the eye blink peak of EOG can be identified.

\section{RESULTS AND DISCUSSION}

The process of EOG analysis is as follows:

1) Denoising. Wavelet Transform was used to remove the high frequency artifacts of EOG signal. The parameter settings of wavelet denoising method are: wavelet base: DB4, decomposition level: 3 . Soft threshold denoising method and unbiased threshold estimation method was adopted.

2) Fuzzy c-means. The EOG signal was divided into two clusters. The eye blink peak was found in the higher amplitude cluster. Figure 1 shows the eye blink peak point of EOG signals. It can be seen that the proposed method can find the eye blink peak correctly. The eye blink peaks for all the EOG signals were detected by using the same method.

3) Feature extraction. The eye blink interval was calculated. Figure 2 shows the mean eye blink interval of 1 minute of SC2. The EBI tends to decrease (increase) when task load increase (decrease). For the purpose of analyzing the relation of EOG to OFS, four features of EBI, i.e. average of EBI (AEBI), variance of EBI (VEBI), standard deviation of EBI (SDEBI) and variation coefficient of EBI (VCEBI), were extracted. The value of each feature in each condition was 
calculated to observe its changes in the whole experiment. Figure 3-6 show the changes of these four features in the whole experiment of SC2. Figure 7 shows the number of subsystems under manual control and subjective measurements in eight control conditions of SC2, respectively.

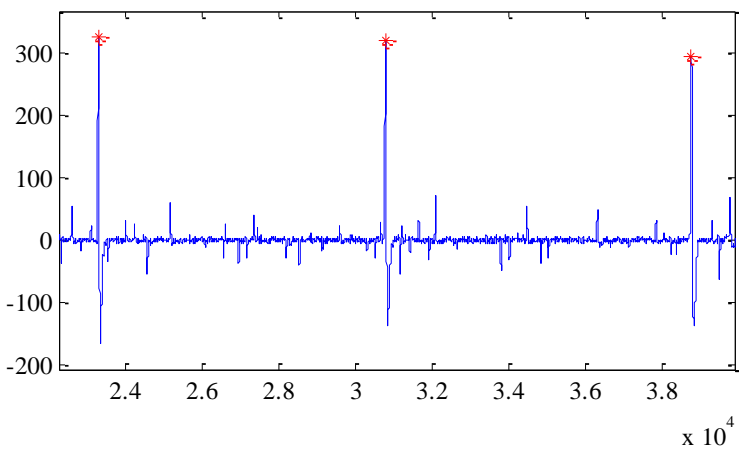

Fig. 1. The results of fuzzy c-means: line-EOG signal;*—eye blink peak point

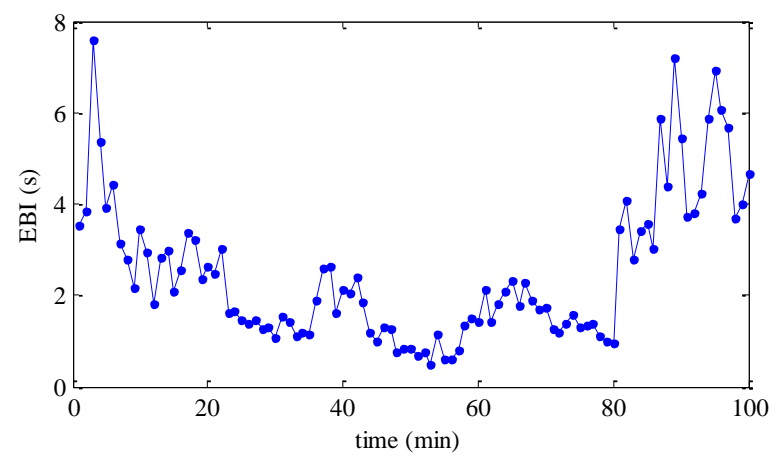

Fig. 2. EBI per minute

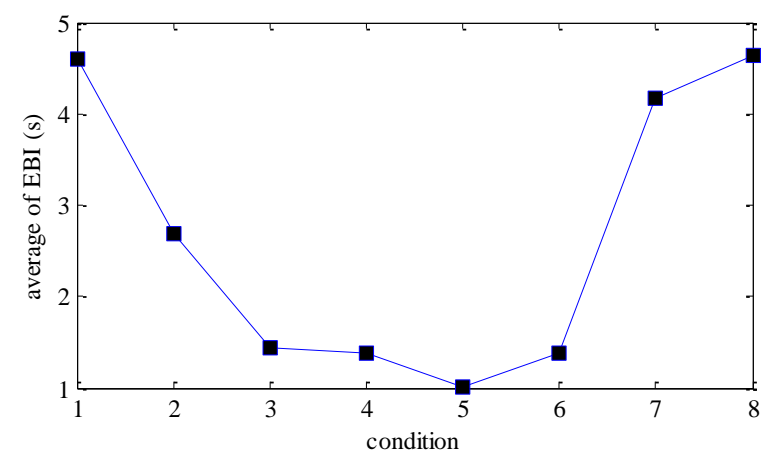

Fig. 3. Average of EBI

The average of EBI, variance of EBI and standard deviation of EBI decrease when the operator's task load increases from $\mathrm{C} 1$ to $\mathrm{C} 4$. And these features show the same changes when the operator's task load decrease from C5-C8. It is clear that the average of EBI, variance of EBI and standard deviation of EBI has a significant relation to operator's task load.

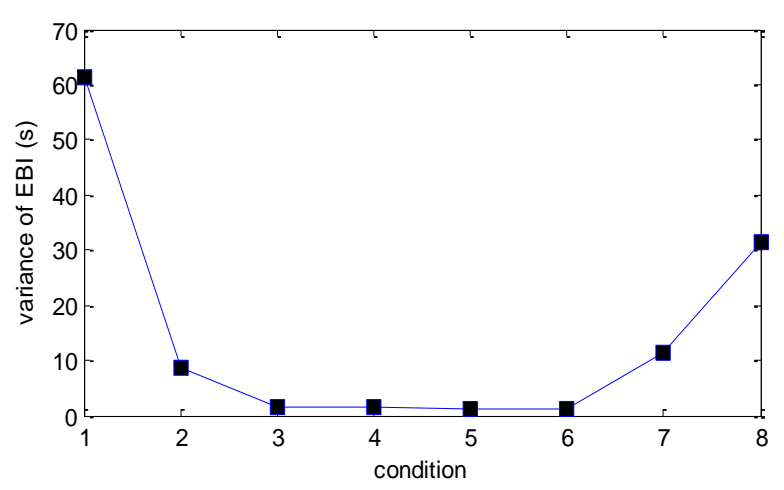

Fig. 4. Variance of EBI

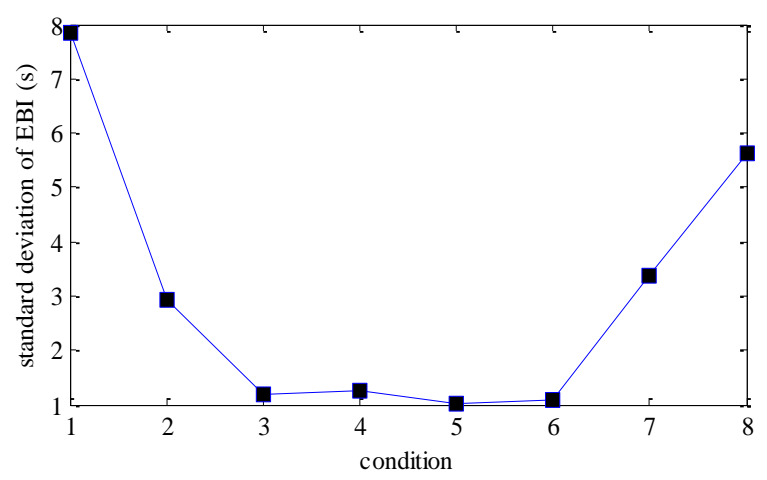

Fig. 5. Standard deviation of EBI

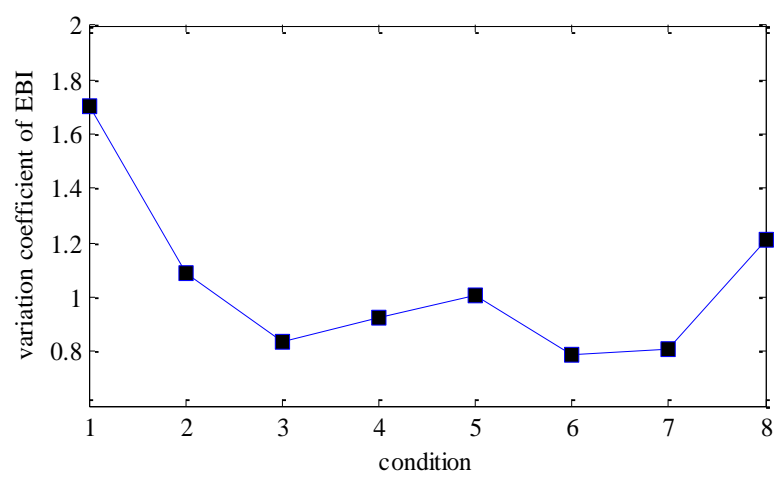

Fig. 6. Variation coefficient of EBI

The variation coefficient of EBI increases from C3-C5, which may be influenced by the operator's increasing effort and anxiety. In C1 and C8, AUTOCAMS runs automatically.

The operator was required to monitor the system. Thus there wasn't any task load for the operator. In C2-C7, the operator needs to control some of the subsystems. From all the four features, it could be seen that there are significant differences between no-load conditions ( $\mathrm{C} 1$ and $\mathrm{C} 8)$ and under load conditions (C2-C7). The difference of features between $\mathrm{C} 1$ and $\mathrm{C} 8$ may be caused by the subject's fatigue. 


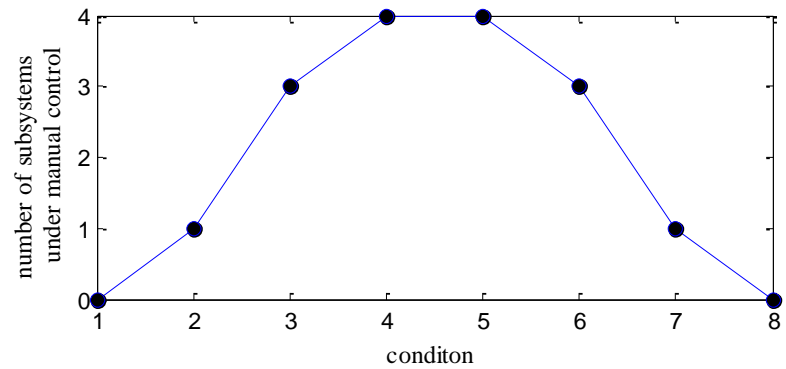

(a)

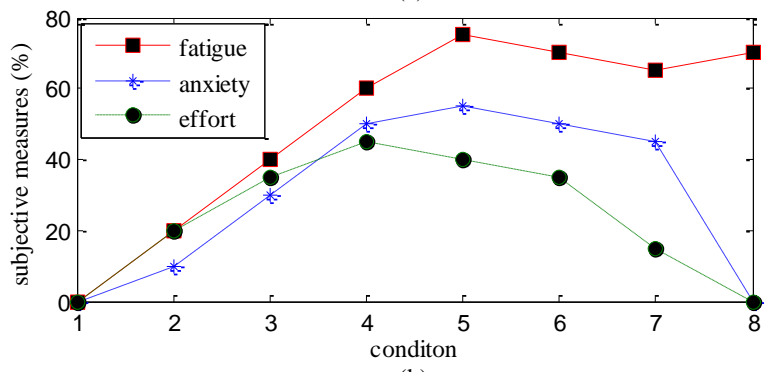

(b)

Fig. 7. The number of subsystems under manual control and subjective measurements

These finds indicate that the changes of task load and mental workload can be reflected by features of EOG. The correlative analysis is used to quantitatively analyze the relationship between EOG features and operator task load, anxiety, effort and fatigue. Table 1 shows the results of correlative analysis between EOG features and operator fatigue, anxiety, effort and task load of SC2. Operator's fatigue can be reflected by variation coefficient of EBI. All these four features can be indices of operator's anxiety. Average of EBI, variance of EBI and standard deviation of EBI have significant relations with operator's effort and task.

TABLE I. CORRELATION ANALYSIS BETWEEN EOG FEATURES AND OPERATOR FUNCTIONAL STATE

\begin{tabular}{|c|l|l|l|l|}
\hline $\begin{array}{c}\text { Operator } \\
\text { Functional } \\
\text { State }\end{array}$ & $\begin{array}{c}\text { Average of } \\
\text { EBI }\end{array}$ & $\begin{array}{c}\text { Variance of } \\
\text { EBI }\end{array}$ & $\begin{array}{c}\text { Standard } \\
\text { Deviation of } \\
\text { EBI }\end{array}$ & $\begin{array}{c}\text { Variation } \\
\text { Coefficient } \\
\text { of EBI }\end{array}$ \\
\hline Fatigue & -0.34 & -0.6 & -0.56 & -0.68 \\
\hline Anxiety & -0.72 & -0.77 & -0.82 & -0.76 \\
\hline Effort & -0.97 & -0.85 & -0.93 & -0.69 \\
\hline Task & -0.95 & -0.78 & -0.89 & -0.62 \\
\hline
\end{tabular}

TABLE II. SIGNIFICANT EOG FEATURES RELATED TO OPERATOR FUNCTIONAL STATE

\begin{tabular}{|l|l|l|l|l|}
\hline No. & Fatigue & \multicolumn{1}{|c|}{ Anxiety } & \multicolumn{1}{c|}{ Effort } & Task load \\
\hline & & SDEBI, & & SDEBI, \\
SA1 & & VCEBI & SDEBI, VCEBI & VCEBI \\
\hline SA2 & & VEBI, SDEBI & SDEBI, VCEBI & VEBI, SDEBI \\
\hline SA3 & & AEBI, SDEBI & AEBI, SDEBI & AEBI, SDEBI \\
\hline & & SDEBI, & & \\
SB1 & VEBI, SDEBI & VCEBI & & \\
\hline SB2 & AEBI,VEBI & & AEBI & \\
\hline SB3 & VEBI, SDEBI & AEBI,VEBI & & \\
\hline SC1 & VEBI,VCEBI & VEBI,VCEBI & VEBI,VCEBI & VEBI, SDEBI \\
\hline SC2 & VEBI,VCEBI & VEBI, SDEBI & AEBI, SDEBI & AEBI, SDEBI \\
\hline SC3 & VEBI,VCEBI & AEBI,VCEBI & AEBI,VCEBI & AEBI,VCEBI \\
\hline
\end{tabular}

The same analysis was done to the EOG signals for all the subjects. Table 2 shows the top 2 significant EOG features $(\mathrm{P}<0.05)$ for each subject. Variance of EBI is a good index of operator fatigue. Standard deviation of EBI can reflect the operator's anxiety, effort and task load. It could be seen that the salient features for different subjects are different owing to individual difference. For subject SA, standard deviation of EBI is a salient feature of operator anxiety, effort and task load. For subject SB, variance of EBI can significantly reflect the changes of operator fatigue. For subject SC, variance of EBI and variation coefficient of EBI are significant features of fatigue and anxiety. Average of EBI can reflect the changes of effort and task load.

\section{CONCLUSION}

This paper analyzed the EOG signal of operator. Fuzzy cmeans was used to detect the eye blink peak of EOG. Average of EBI, variance of EBI, standard deviation of EBI and variation coefficient of EBI were extracted. The relation of EOG features and operators fatigue, anxiety, effort and task load was analyzed. Salient EOG features for each subject are successfully extracted. The experiment results show that it's necessary to assess the operator functional state using personal salient features. The results of this paper could be used to identify operator functional sate in future works. Future works will focus on two areas. First, the authors will do more experiments and further analysis on EOG, in order to evaluate the individual differences. Second, models that describe the relation between EOG features and operator functional state will be constructed.

\section{ACKNOWLEDGMENTS}

This work is partially supported by the Shanghai University Young Teachers' Training Scheme Funds ZZGJD12011, the National Nature Science Foundation under Grant No. 61403249 and No. 61403248.

\section{REFERENCES}

[1] G. Borghini, L. Astolfi, G. Vecchiato, D. Mattia, F. Babiloni, "Measuring neurophysiological signals in aircraft pilots and car drivers for the assessment of mental workload, fatigue and drowsiness", Neurosci. Biobehav. R. Kidlington, vol. 44, pp. 58-75, July 2014.

[2] R. Wang, J. Zhang, Y. Zhang, X. Wang, “Assessment of human operator functional state using a novel differential evolution optimization based adaptive fuzzy model", Biomed. Signal Process. Control, Kidlington, vol. 7, pp. 490-498, September 2012.

[3] K. L. Saroj, C. Ashley, "Driver fatigue : electroencephalography and psychological assessment", Psychophysiol. Malden MA, vol. 39, pp. 313-321, May 2002.

[4] I. Kathner, S. C. Wriessnegger, G. R. Muller-Putz, A. Kubler, S. Halder, "Effects of mental workload and fatigue on the P300, alpha and theta band power during operation of an ERP (P300) brain-computer interface", Biol. Psychol. Amsterdam, vol. 102, pp. 118-129, October 2014.

[5] S. Rodriguez-Ruiz, P. M. Guerra, S. Moreno, M. Carmen Fernandez, J. Vila, "Heart rate variability modulates eye-blink startle in women with bulimic symptoms", J. Psychophysiol. Gottingen, vol. 26, pp. 10-19, October 2012.

[6] H. A. Slagter, K. Georgopoulou, M. J. Frank, "Spontaneous eye blink rate predicts learning from negative, but not positive, outcomes", Neuropsychologia, Kidlington, vol. 71, pp. 126-132, May 2015.

[7] M. J. Doughty, "Further assessment of gender- and blink patternrelated differences in the spontaneous eyeblink activity in primary gaze in 
young adult humans", Optometry Vision Sci. Philadelphia, vol. 79, pp. 439-447, July 2002.

[8] P. L. Kaufman, A. ALM, "Adler's physiology of the eye", 10th ed. St. Louis: Mosby, 2003.

[9] M. A. Recarte, L. M. Nunes, "Mental workload while driving: effects on visual search, discrimination, and decision making", J. Exp. Psychol. Appl. Washington, vol. 9, no. 2, pp. 119-137, June 2003.

[10] M. Boyer, M. L. Cummings, L. B. Spence, E. T. Solovey, "Investigating mental workload changes in a long duration supervisory control task", Interact. Comput. Oxford, May 2015.

[11] G. R. J. Hockey, D. G. Wastell, J. Sauer, "Effects of sleep deprivation and user-interface on complex performance: a multilevel analysis of compensatory control”, Human Factors. vol. 40, no. 2, pp. 233-253, June 1998.

[12] B. Lorenz, F. DiNocera, S. Rottger, R. Parasuraman, "Automated faultmanagement in a simulated spaceflight micro-world", Aviat. Space Environ. Med. Washington, vol. 73, 886-897, September 2002.

[13] J.C. Bezdek, "Pattern recognition with fuzzy objective function algorithms", New York: Plenum Press, 1981.

[14] J. C. Bezdek, Pattern Recognition with Fuzzy Objective Function Algorithms. Springer Science \& Business Media, 2013.

[15] N. R. Pal, K. Pal, J. M. Keller, J. C. Bezdek, "A possibilistic fuzzy cmeans clustering algorithm", IEEE Trans. Fuzzy Syst. vol. 13, pp. 517530, August 2005. 